\title{
Factores explicativos en la calidad de servicio de las salas de cine
}

\author{
Explanatory factors in the quality \\ of service in the cinemas
}

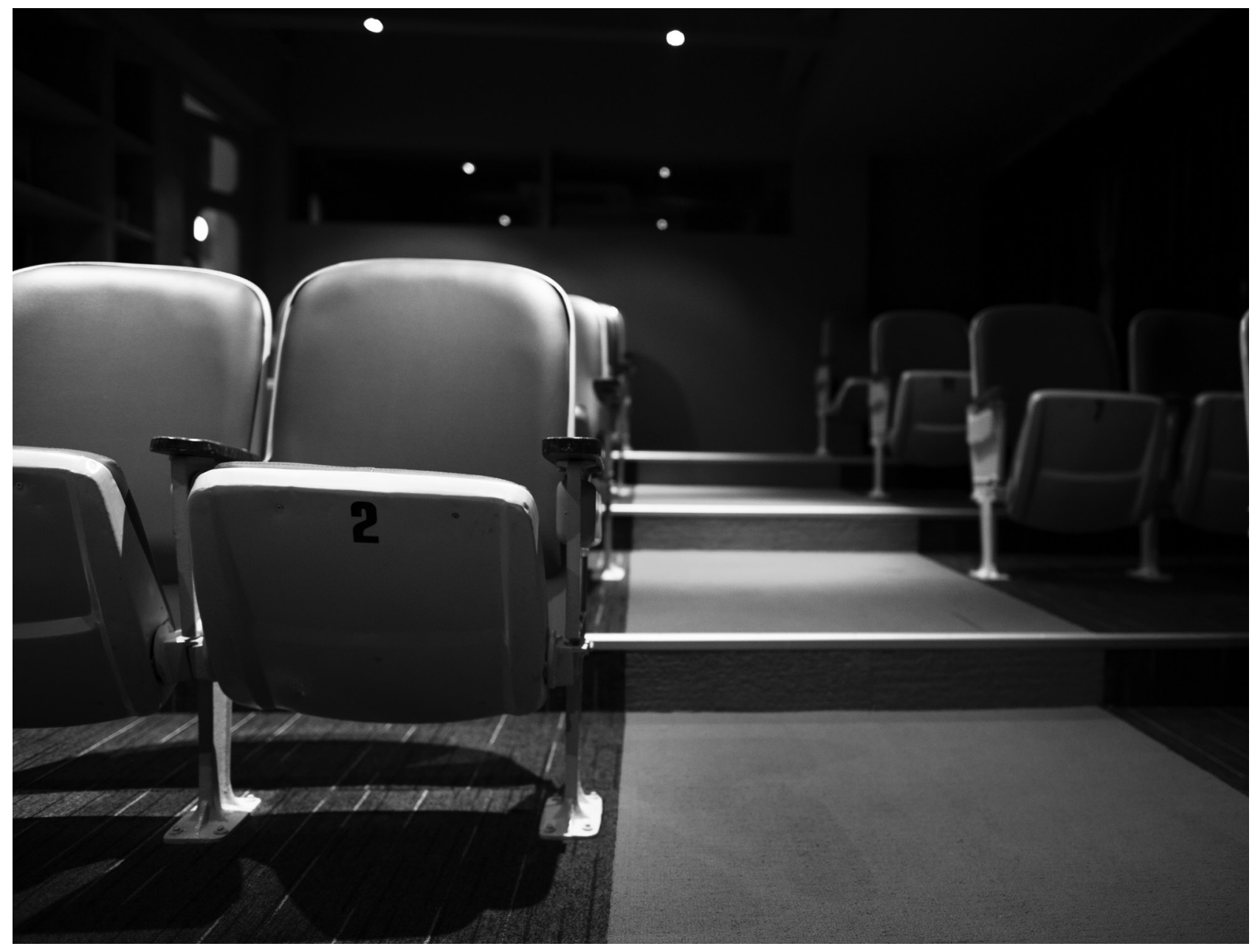




\title{
Factores explicativos en la calidad de servicio de las salas de cine ${ }^{1}$
}

\section{Explanatory factors in the quality of service in the cinemas}

\author{
Domingo José Martínez Díaz², David Eduardo Juliao Esparragoza ${ }^{3}$
}

Artículo recibido en agosto de 2017; artículo aceptado en septiembre de 2017.

Este artículo puede compartirse bajo la Licencia Creative Commons Atribución-No Comercial-Compartir lgual 2.0 Genérica y se referencia usando el siguiente formato: Martínez, D. \& Juliao, D. (2018). Factores explicativos en la calidad de servicio

de las salas de cine. I+D Revista de Investigaciones, 11(1), 93-104. DOI: https://doi.org/10.33304/revinv.v11n1-2018008

\begin{abstract}
Resumen
El estudio es un diseño experimental que explica si los factores día de la película y tipo de sala influyen en la percepción de la calidad de las salas de cine; para evaluar la percepción de los elementos de la calidad de un servicio se utilizó una escala. El cine es una de las alternativas más frecuentadas de consumo de ocio en Colombia (DANE); la importancia del estudio es contribuir con información para que las salas de cine puedan tomar decisiones asertivas que les permitan implementar estrategias que respondan a los elementos claves que influyen en la satisfacción esperada por la demanda. Con un nivel de significancia del $5 \%$, se descubrió que las dimensiones tangibilidad y capacidad de respuesta influyen en la percepción de la calidad del servicio de cine. Los hallazgos le permiten a la gerencia de las salas administrar discrecionalmente los factores influyentes en la calidad percibida.
\end{abstract}

Palabras clave: método experimental, estudio de mercado, diseño factorial, cine, calidad percibida.

\begin{abstract}
This study is an experimental design that explains if the day factors of the film and type of room influence the perception of the quality of the cinemas. A scale was used to evaluate the perception of the service quality elements. The cinema is one of the most frequent alternatives of leisure consumption in Colombia (DANE), the importance of the study is to contribute with information to that cinemas can make assertive decisions so that they implement strategies that respond to the key elements that they influence the satisfaction expected by the demand. With a level of significance of $5 \%$, it was discovered that the dimensions of tangibility and response capacity influence the perception of the quality of the cinema service. The findings allow the management of the rooms to discretionally administrate the influential factors in the perceived quality.
\end{abstract}

Keywords: experimental method, market study, factorial design, cinema, perceived quality.

\footnotetext{
1. Artículo de investigación, con diseño experimental, resultado de un proyecto de investigación de mercados, perteneciente al área de marketing. Desarrollado en el Grupo de Investigación en Marketing -GIM. Universidad del Norte, Barranquilla- Colombia. Dirección: Km 5 Vía Puerto Colombia; PBX: +57 (5) 3509509. Fecha de inicio: enero 2017, fecha de terminación: junio 2017.

2. Estadístico, Universidad de la Salle. Doctor en Psicología, Universidad del Norte. Docente- investigador del grupo GIM, Universidad del Norte, Barranquilla (Colombia): Dirección: Km 5 vía puerto Colombia, PBX: (+57) (5) 3509509. ORCID ID: https://orcid.org/0000-0002-3522-5379. Correo institucional: domimart@uninorte.edu.co.

3. Economista, Universidad del Atlántico. Magíster en Administración de Empresas, Universidad del Norte. Docente- investigador del grupo GIM, Universidad del Norte, Barranquilla (Colombia). Dirección: Km 5 vía puerto Colombia, PBX: (+57) (5) 3509509. ORCID ID: https://orcid.org/0000-0002-41423873. Correo institucional: djuliae@uninorte.edu.co.
} 


\section{Introducción}

En Colombia, son pocos los documentos que intentan describir el impacto del cine como actividad económica. Uno de los primeros intentos fue el de Jaramillo, Reina y Zuleta (2003), quienes pudieron, a través de información primaria, crear un "estado del arte" del impacto de esta actividad en la economía. El cine tiene en sí mismo una serie de negocios conexos y amalgamados que componen su cadena de valor, siendo los principales la producción y la exhibición.

El negocio del cine movió en Colombia, según cifras de la firma Raddar, 364000 millones de pesos en 2013 y ha tenido inversiones en los últimos tres años más de 35 millones de dólares en las nuevas salas de exhibición (Iglesias, 2014). De acuerdo con la encuesta sobre consumo cultural en Colombia (ECC), el $35 \%$ de los encuestados manifestó haber ido al cine por lo menos una vez en los últimos doce meses, lo cual muestra esta alternativa de entretenimiento cultural como la más frecuentada por el mercado colombiano (Departamento Administrativo Nacional de Estadística - DANE, 2016).

En términos agregados, según la ECC la asistencia a cine en Colombia aumentó, pasando de $33,8 \%$ en 2014 a $40,2 \%$ en 2016. En la zona Atlántica la variación bienal registrada (2014-2016) es de 2,3\% (DANE, 2016).

La encuesta refleja, entre otras cosas, las preferencias por tipo de película, cuánto se pagó por entrar a cine y la frecuencia promedio de visitas, entre otras. Una de las preguntas formuladas en la encuesta es la relacionada con las razones por las cuales no se va a cine. En esta pregunta, una de las opciones de respuesta era la ausencia de proyecciones especiales o salas de cine, un insumo a nuestro parecer importante para los negocios de proyección (salas de cine), pues ofrece una visión de la percepción del cliente final en términos del canal. No se aprecian en el análisis de la ECC las preferencias del usuario por tipologías de salas y es ahí donde esta investigación puede aportar a una industria dinámica al servicio del entretenimiento para empezar a entender si esta variable influye o no en la calidad percibida de la experiencia.

Para descubrir si la variable tipo de sala influye en la calidad percibida del cine se utilizó el diseño de experimentos, el cual se asocia en investigación de mercados a los diseños causales (Malhotra, 2007). Se entiende causalidad desde la perspectiva científica, donde los hallazgos no son respuestas fácticas sino probables (Martínez \& Tordera, 1995; McDaniel \& Gates, 2015).

De acuerdo con Malhotra (2007), los diseños experimentales pueden clasificarse en preexperimentales, experimentales verdaderos, cuasi experimentales y estadísticos. Normalmente, se hacen para estimar efectos en las variables de interés (Pascual, García \& Frías, 1996).

Como técnicas más importantes en relación con los diseños estadísticos se utilizan el diseño de bloques aleatorizados, el cuadrado latino y el diseño factorial. Los métodos buscan el control de las variables externas y verificar la probabilidad de influencia en una variable dependiente.

Una de las ventajas de los experimentos factoriales es que sirven para determinar si cada factor ejerce alguna acción sobre la variable respuesta, obteniendo un cuadro amplio de los efectos de los factores (Chacín, 2000).

La herramienta estadística utilizada en estos casos es el análisis de varianza, el cual debe aplicarse observando no incurrir en errores frecuentes al comparar poblaciones independientes (Díaz, 2009).

Se consideran en el experimento las condiciones vitales de causalidad: variación concomitante, ordenanza de hechos y disminución de otros factores causales posibles (Ato, López \& Benavente, 2013).

En el modelo estadístico de los diseños factoriales con dos factores A y B se afirma que el comportamiento de la variable dependiente o respuesta $Y$ en el experimento con K réplicas, se podrá describir mediante el modelo de efectos definido por Gutiérrez y De la Vara (2004, p.158), así:

$Y i j k=\mu+a i+\beta j+(a \beta) i j+\varepsilon i j k ; i=1,2, \ldots, b ; k=1,2, \ldots, n$

Donde $\mu$ es la media general, a es el efecto debido al i-simo nivel del factor $A, \beta$ es el efecto del j-ésimo nivel del factor $B, a \beta$ representa al efecto de interacción en la combinación ij y $\varepsilon$ es el error aleatorio que supone sigue una distribución normal con media cero y varianza constante y son independientes entre sí.

Por otra parte, y teniendo en cuenta que el estudio cobija la calidad de servicio y el involucramiento de estudiantes en la recolección de datos, es necesario revisar los enfoques teóricos sobre la satisfacción y la calidad percibida.

Folgueiras, Luna \& Puig (2013) manifiestan que la interacción de estudiantes cuando se involucran en actividades comunitarias para su aprendizaje aumenta su rendimiento y genera mella en su cognición, además, el ejercicio puede servir para construir alternativas de mejora en su proceso. Este hallazgo puede leerse como una forma práctica y económica de buscar procesos de cocreación de valor entre docentes y dicentes con el propósito de encontrar rutas alternativas y vinculantes 
del proceso de aprendizaje. Asimismo, Barberà y Valdés (1996); Machado, Montes \& Mena (2008) coinciden en que la metodología de enseñanza y la forma de evaluación pueden conducir a dominios cognitivos diferentes. Se usa como referente que tareas que involucren alta participación del estudiante están en una escala mayor en la taxonomía de Bloom (García \& Amante, 2006).
En relación con la calidad de servicio, se puede mencionar que el término tiene varias acepciones (Saren, 2006). Se puede verificar el concepto de calidad como mediada desde la ingeniería aduciendo las capacidades técnicas medibles y comprobables, pero también se puede tener en cuenta el concepto de calidad percibida como medida de control. Algunas aproximaciones al concepto de calidad se resumen en la Tabla 1.

Tabla 1

Listado de autores sobre el concepto de calidad

\begin{tabular}{|c|c|c|}
\hline Año & Autor & Definición \\
\hline 1988 & Crosby & $\begin{array}{l}\text { "De manera sintética, calidad significa calidad del producto. Más específicamente, calidad es calidad } \\
\text { del trabajo, calidad del servicio, calidad de la información, calidad de proceso, calidad de la gente, } \\
\text { calidad del sistema, calidad de la compañía, calidad de objetivos, etc.". }\end{array}$ \\
\hline 1989 & Deming & $\begin{array}{l}\text { "Calidad es conformidad con los requerimientos. Los requerimientos tienen que estar claramente } \\
\text { establecidos para que no haya malentendidos; las mediciones deben ser tomadas continuamente para } \\
\text { determinar conformidad con esos requerimientos; la no conformidad detectada es una ausencia de } \\
\text { calidad". }\end{array}$ \\
\hline 1990 & Juran & $\begin{array}{l}\text { "1) La calidad consiste en aquellas características de producto que se basan en las necesidades del } \\
\text { cliente y que por eso brindan satisfacción del producto. 2) Calidad consiste en libertad después de las } \\
\text { deficiencias". }\end{array}$ \\
\hline 1990 & Drucker & $\begin{array}{l}\text { "La calidad no es lo que se pone dentro de un servicio, es lo que el cliente obtiene de él y por lo que } \\
\text { está dispuesto a pagar". }\end{array}$ \\
\hline 1998 & Imai & $\begin{array}{l}\text { "La calidad se refiere, no solo a productos o servicios terminados, sino también a la calidad de los } \\
\text { procesos que se relacionan con dichos productos o servicios. La calidad pasa por todas las fases de la } \\
\text { actividad de la empresa, es decir, por todos los procesos de desarrollo, diseño, producción, venta y } \\
\text { mantenimiento de los productos o servicios". }\end{array}$ \\
\hline
\end{tabular}

Fuente: Tomado de Duque (2005) p. 67-68.

Para el análisis se tienen en cuenta tres actores fundamentales: agentes, relaciones y entorno. Asimismo, son parte de la perspectiva de abordaje la calidad objetiva y subjetiva, esta última con implicaciones profundas en torno a la percepción de valor y aplicaciones en mercadeo.

De acuerdo con Duque (2005), la revisión de calidad objetiva subyace a parámetros estadísticos, es verificable a nivel técnico y está sujeta a revisiones internas. La calidad subjetiva, por su parte, tiene que ver con la visión externa del proceso en términos de las expectativas de los clientes. La calidad percibida entonces se refiere "al juicio del consumidor sobre la excelencia y superioridad de un producto" (Zeithaml, 1988, Citado por Duque, 2005).

Dadas las características implícitas en la parametrización de la medición de la calidad subjetiva es importante mencionar que esta tiene rasgos multidimensionales y actúa en concordancia con el manejo de percepciones.
Es importante tener en cuenta que la calidad de los servicios es compleja de medir y esta medición se considera fugaz (Parasuraman, Zeithaml \& Berry, 1988). Rust y Oliver (1994), citados por Duque (2005), plantearon como punto de partida que la calidad de servicio percibida dependía de la comparación del servicio esperado con el servicio percibido.

Por su parte, Sureshchandar, Rajendran y Kamalanabhan (2001) analizaron el concepto no solo desde los elementos tangibles sino también desde las interacciones humanas. Sin embargo, pasaron poralto otros factores de suma importancia como productos, sistematización y estandarización (Berdugo, Barbosa \& Prada, 2016).

Otras teorías sobre el concepto de calidad manejan cinco dimensiones (Parasuraman, Berry \& Zeithaml, 1992): elementos tangibles, fiabilidad, capacidad de respuesta, seguridad y empatía. 
Así las cosas, diferentes autores establecieron parámetros o dimensiones sobre las que reposaba el constructo de calidad percibida. Las diferentes perspectivas, entre las cuales se destacan la de Grönroos (1988) y Grönroos, Storbacka y Strandvik (1994), donde explica cómo esta definición involucra el juicio subjetivo del cliente sobre el servicio que recibe (Losada \& Rodríguez, 2007), merecen especial atención. Por su parte, Parasuraman, Zeithaml y Berry (1985) convergieron en modelos de medición posteriores, como los que se registran en la Tabla 2.

Tabla 2

Conceptos de calidad percibida

\begin{tabular}{|c|c|c|}
\hline Modelo & Autor & Definición \\
\hline $\begin{array}{l}\text { Modelo de la } \\
\text { imagen (Escuela } \\
\text { Nórdica) }\end{array}$ & $\begin{array}{l}\text { Grönroos } \\
(1988,1994)\end{array}$ & $\begin{array}{l}\text { La calidad percibida por los clientes es la integración de la calidad técnica (qué } \\
\text { se da) y la calidad funcional (cómo se da), y estas se relacionan con la imagen } \\
\text { corporativa. La imagen es un elemento básico para medir la calidad percibida. }\end{array}$ \\
\hline $\begin{array}{l}\text { Modelo ServQual } \\
\text { (Escuela } \\
\text { Americana) }\end{array}$ & $\begin{array}{l}\text { Parasuraman, } \\
\text { Zeithaml y Berry } \\
(1985,1988)\end{array}$ & $\begin{array}{l}\text { Medición a través de la evaluación por separado de las expectativas y } \\
\text { percepciones de un cliente, apoyándose en los comentarios hechos por los } \\
\text { consumidores en la investigación. Estos comentarios apuntan hacia diez } \\
\text { dimensiones establecidas por los autores y con una importancia relativa que, } \\
\text { afirman, depende del tipo de servicio o cliente. }\end{array}$ \\
\hline $\begin{array}{l}\text { Modelo ServQual } \\
\text { (Escuela } \\
\text { Americana) }\end{array}$ & $\begin{array}{l}\text { Parasuraman, } \\
\text { Zeithaml y Berry } \\
\text { (1993) }\end{array}$ & $\begin{array}{l}\text { Después de un análisis estadístico los autores reducen las dimensiones a cinco } \\
\text { factores: elementos tangibles, fiabilidad, capacidad de respuesta, garantía y } \\
\text { empatía. }\end{array}$ \\
\hline $\begin{array}{l}\text { Modelo de los } \\
\text { tres componentes }\end{array}$ & $\begin{array}{l}\text { Rust \& Oliver } \\
(1994)\end{array}$ & $\begin{array}{l}\text { El ambiente del servicio está subdividido en dos perspectivas: la interna (del } \\
\text { proveedor del servicio) y la externa. El ambiente interno se enfoca en la cultura } \\
\text { organizacional y en la filosofía de la eliminación, mientras el externo se orienta } \\
\text { principalmente al ambiente físico de la prestación del servicio. }\end{array}$ \\
\hline
\end{tabular}

Fuente: Tomado de Duque (2005) y aportes propios.

Teniendo en cuenta estas características es posible abordar la calidad percibida desde diferentes metodologías; en el caso de este estudio, se utilizó la herramienta ServQual como instrumento validado y aceptado (Mejías, 2005).

Volviendo al cine, este negocio se incluye en el mercadeo de servicios porque el producto que ofrece es un servicio que se define como un conjunto de acciones, actuaciones y desempeños que se diferencian de los bienes físicos especialmente por sus características de intangibilidad (no se puede tocar), heterogeneidad (variabilidad de comportamientos), inseparabilidad (compra y consumo simultáneo) y caducidad (no se almacena), aunque estas características no son exclusivas de todos los servicios (Giraldo, 2016).

Según Fisky Tansuhaj (1985), citados por Giraldo (2016), el cine forma parte de los servicios de entretenimiento junto con servicios tales como deportes de automovilismo, ciclismo, béisbol, o servicios como son las artes de ópera y teatro o también servicios.

\section{Método}

\section{Tipo de estudio}

El método de investigación es experimentación. Se hizo un experimento de campo que buscaba medir la calidad del servicio de cine en función de dos factores o variables como son el día en que se exhibe la película y el tipo de sala que el consumidor decide para ver la cinta. Para esta investigación se utilizó un diseño de dos factores, con 3 y 6 niveles de tratamiento para cada factor. Los dos factores se establecieron como las variables independientes o explicativas. El factor día de la semana se concibe como un factor cualitativo específico, ya que sus niveles no siguen un orden natural y cada nivel es de igual interés; por su parte, el factor tipo de sala es un factor cualitativo ordenado porque sus niveles de factor están arreglados en algún orden, pero no hay una variable cuantitativa natural que describa sus niveles, por lo que los diferentes niveles no corresponden a valores bien definidos sino más bien a valores agrupados (Chacín, 2000).

\section{- Factor o Variable 1: día de la película.}

Esta variable se define como los días de la semana durante los cuales la industria del cine ha programado la presentación de las películas en sus salas de cine. Este factor se clasifica en tres tratamientos:

Tratamiento 1: lunes-martes-jueves 
Tratamiento 2: miércoles

Tratamiento 3: fin de semana

Se escoge el día de la película toda vez que los tratamientos están estrechamente relacionados con las estrategias para normalizar la demanda, haciendo que los distribuidores en días valle (poca demanda) generen incentivos para que el público asista (Restrepo, 2007; Nagle, Tagle \&Holden, 2002). En este caso, los días de mayor demanda son los fines de semana, y el miércoles se ofrece promoción de boletas a mitad precio.

\section{- Factor o variable 2: Tipo de sala.}

En este factor, la industria del cine ofrece al mercado la siguiente variedad de salas de cine:

Tratamiento 1: sala 2D general

Tratamiento 2: sala 2D preferencial

Tratamiento 3: sala 3D general

Tratamiento 4: sala 3D preferencial

Tratamiento 5: sala 4D

Tratamiento 6: sala VIP

Se escoge el tipo de sala toda vez que los elementos que la componente intervienen directamente en la percepción que tiene el usuario sobre la película. Se encuentra documentado que existen diferentes formas de acercarse al cine en lo que Artopoulos (2011) denomina las cuatro pantallas: la pantalla grande de los cines, la televisión, la computadora y el celular. El punto clave ya no es la distinción sino la interacción que hace que el cine en salas sea apreciado como un producto diferenciado.

En este sentido, la sala 3D ha ofrecido desde su aparición una experiencia única que, junto con los efectos especiales, se unen a un fenómeno denominado "nuevo cine de espectáculo" por su apelación preferencial a la experiencia sensorial (Moguillansky, 2016). Otros aspectos destacados que ofrecen distinción en las salas son la comodidad en términos de espacio y lejanía de la pantalla, por una parte, y por otra, el servicio al sillón y ergonomía sofisticada, esto en los tratamientos 2D, 3D preferencial y VIP respectivamente.

\section{- Variable dependiente}

\section{Calidad del servicio}

Corresponde a la variable respuesta que se midió en el experimento. La calidad del servicio es una variable métrica que se midió siguiendo el modelo Servqual.

\section{Participantes}

Participaron un total de 84 sujetos, estudiantes de sexto a décimo semestre de Administración de Empresas de la Universidad del Norte de Barranquilla. Las características demográficas se discriminan en la Tabla 3.

\section{Tabla 3}

Características de la muestra según género y edad

\begin{tabular}{ccccc}
\hline & & \multicolumn{2}{c}{$\begin{array}{c}\text { Género del } \\
\text { estudiante }\end{array}$} & \\
\cline { 3 - 4 } & & Mujer & Hombre & Total \\
\hline & $\begin{array}{c}\text { Máximo 19 años } \\
\text { Entre 19 y 21 } \\
\text { años }\end{array}$ & 26 & 27 & 53 \\
Edad del & $\begin{array}{c}\text { Entre 21 y 23 } \\
\text { estudiante }\end{array}$ & 7 & 15 & 22 \\
& $\begin{array}{c}\text { Entre } 23 \text { y } 25 \\
\text { años }\end{array}$ & 1 & 2 & 3 \\
& Más de 25 años & 0 & 1 & 1 \\
\hline & Total & 35 & 49 & 84 \\
\hline
\end{tabular}

Fuente: Propia a partir de los resultados obtenidos mediante SPS.

ElmodeloServQual (Parasuraman, Zeithaml\&Berry, 1993) es una herramienta que permite medir la calidad del servicio de una organización. Los autores de este modelo desarrollaron un instrumento que permitió medir la percepción de los usuarios sobre la calidad de los servicios que consumen. Además, el instrumento está validado estadísticamente y tiene aceptación general. Sus validaciones psicométricas pueden evidenciarse (Parasuraman, Berry \& Zeithaml, 1991). Un servicio de calidad se define cuando existe un balance positivo entre lo que se espera y lo que se obtiene (diferencia entre las expectativas y percepciones de los clientes) (Marzo, Martínez, Ramos \& Peiró, 2002).

Cabe señalar que existen ciertos factores clave que condicionan las expectativas de los usuarios. En este sentido, los autores le dan importancia a la comunicación "boca a oreja" (Molla, Eiglier \& Langeard, 1989), a las opiniones y recomendaciones de amigos y familiares sobre el servicio (Anzures, 2013; Mesa, 2005), a las necesidades personales, a las experiencias con el servicio que el usuario haya tenido con anterioridad, e, igualmente a las comunicaciones externas que la propia empresa realice sobre las prestaciones de su servicio y que inciden en las expectativas que las personas no tienen sobre estas.

Las cinco dimensiones identificadas como criterios de evaluación por parte de los consumidores para valorar la calidad en un servicio, se definen así:

- Elementos tangibles. Apariencia de las instalaciones 
físicas, equipos, personal y materiales de comunicación.

- Fiabilidad. Habilidad para realizar el servicio de modo cuidadoso y fiable.

- Capacidad de respuesta. Disposición y voluntad para ayudar a los usuarios y proporcionar un servicio rápido.

- Garantía. Conocimientos y atención mostrados por los empleados y sus habilidades para concitar credibilidad y confianza.

- Empatía. Atención personalizada que dispensa la organización a sus clientes.

\section{Observación natural}

Para asegurar una buena evaluación de los factores investigados, los estudiantes llegaron con anterioridad a la función, reclamaron o compraron los tiquetes en la taquilla y después se dirigieron a la confitería para evaluar la calidad y precios de los productos que se ofrecían en este lugar; seguidamente, observaron si los productos ofrecidos eran los suficientemente variados para satisfacer al público en general.

Asimismo, chequearon los baños, qué tan limpios y en buen estado estaban para el uso del público. Adicionalmente, durante la función tuvieron en cuenta la calidad de la proyección, sin perder de vista la comodidad y el espacio en las sillas, todo esto en calidad exclusiva de observadores.

Ingresando ya al análisis y verificación de las hipótesis, se sigue el modelo ServQual evaluando cada una de las cinco características de la calidad del servicio y se termina con la calidad del servicio en general.

En la implementación de este modelo se utilizó un formato tipo cuestionario, el cual fue diligenciado por la unidad de prueba, en este caso el estudiante universitario.

\section{Procedimiento}

Los estudiantes fueron las unidades de prueba, y se organizaron de manera aleatoria de acuerdo con el tipo de sala (2D General, 2D Preferencial, 3D General, 3D Preferencial, 4D y VIP) y día de la semana (lunes- martesjueves o miércoles o fin de semana). El experimento se realizó durante la semana que va del miércoles 3 de mayo al jueves 11 de mayo de 2017. La unidad de prueba decidió la hora y la película que iba a ver, así como el centro comercial donde estaba el tipo de sala asignado. El $67 \%$ de los estudiantes decidieron el Centro Comercial Buenavista porque cuenta con casi todos los tipos de sala. De otra parte, el $23 \%$ de los estudiantes escogieron los centros comerciales Viva y Villa Country porque son los únicos que tienen las salas VIP.

\section{Resultados}

Las dimensiones de la calidad de servicio fueron evaluadas por los participantes del experimento y los investigadores definieron las siguientes hipótesis de investigación:

\section{Hipótesis de trabajo 1}

El factor “Día de la película" es la causa de la percepción que tiene el estudiante universitario frente a la calidad de cada una de las características "Tangibilidad", "Confiabilidad", "Capacidad de Respuesta", "Garantía" y "Empatía" para el servicio que prestan las salas de cine.

\section{Hipótesis de trabajo 2}

El factor "Tipo de sala" es la causa de la percepción del estudiante universitario frente a la calidad de cada una de las características "Tangibilidad", "Confiabilidad", "Capacidad de Respuesta", "Garantía" y "Empatía" para el servicio que prestan las salas de cine.

\section{Hipótesis de trabajo 3}

El factor "Día de la película" es la causa de la percepción del estudiante universitario frente a la calidad del servicio en general que prestan las salas de cine.

\section{Hipótesis de trabajo 4}

El factor "Tipo de sala" es la causa de la percepción del estudiante universitario frente a la calidad del servicio en general que prestan las salas de cine.

\section{Hipótesis de trabajo 5}

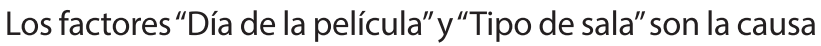
de la percepción del estudiante universitario frente a la calidad de cada una de las características: "Tangibilidad", "Confiabilidad", "Capacidad de Respuesta", "Garantía" y "Empatía" en el servicio que prestan las salas de cine.

\section{Hipótesis de trabajo 6}

Los factores "Día de la película" $y$ "Tipo de sala" son la causa de la percepción del estudiante universitario frente a la calidad del servicio en general que prestan las salas de cine.

\section{Análisis descriptivo}

Este análisis se realiza con el fin de dar lectura al 
comportamiento general del resultado de cada dimensión del modelo ServQual por tipo de sala de cine escogido. Se relacionan las medias de las variables a continuación:

Se toma como punto de referencia que cualquier variable que esté mínimo con un promedio de cuatro se considera que alcanza un buen comportamiento a favor de la calidad percibida; de lo contrario, se recomienda que se desarrollen acciones que mejoren el respectivo componente. En la Tabla 4 se encuentran los resultados de la media y la desviación estándar para cada uno de los elementos del servicio y el servicio en general.

Para todos los tipos de sala los componentes tangibilidad, garantía y empatía alcanzan niveles satisfactorios. Por su parte, el componente confiabilidad muestra aspectos a mejorar en las salas 2D preferencial, 3D General y 4D $(3,9$; 3,8 y 3,8 respectivamente) y la capacidad de respuesta en todas las salas de cine excluyendo VIP muestran los niveles más críticos en aspectos por mejorar con promedios que están por debajo de cuatro.

Teniendo en cuenta los valores encontrados por debajo del nivel esperado tenemos que en las salas $2 \mathrm{D}$ preferencial, 3D General y 4D existe una percepción de la calidad del servicio por debajo de lo esperado, en concreto, en el componente fiabilidad. Lo anterior puede ser explicado en términos de mayores expectativas por precio pagado, en particular, puede deberse a la precisión horaria con que se espera se proyecte la película. Tiempos promedio de espera más largos hacen que la experiencia no sea la esperada (Torres, 2010).

Tabla 4

Media y desviación estándar de la percepción de la calidad del cine

\begin{tabular}{|c|c|c|c|c|c|c|c|}
\hline \multicolumn{2}{|c|}{ Tipo de sala } & \multirow{2}{*}{$\begin{array}{c}\text { Tangibilidad } \\
4,4531\end{array}$} & \multirow{2}{*}{$\begin{array}{c}\text { Confiabilidad } \\
4,0500\end{array}$} & \multirow{2}{*}{$\begin{array}{c}\text { Capacidad de } \\
\text { respuesta }\end{array}$} & \multirow{2}{*}{$\begin{array}{c}\text { Garantía } \\
4,2656\end{array}$} & \multirow{2}{*}{$\begin{array}{c}\text { Empatía } \\
4,3750\end{array}$} & \multirow{2}{*}{$\begin{array}{c}\begin{array}{c}\text { Calidad del } \\
\text { servicio de } \\
\text { cine }\end{array} \\
4,1975 \\
\end{array}$} \\
\hline $2 \mathrm{~d}$ general & Media & & & & & & \\
\hline & $\mathrm{N}$ & 16 & 16 & 16 & 16 & 16 & 16 \\
\hline & $\begin{array}{l}\text { Desviación } \\
\text { estándar }\end{array}$ & ,55692 & ,75366 & 81074 & ,66124 & 74789 & 60195 \\
\hline & Media & 4,2969 & 3,9000 & 3,7656 & 4,1563 & 4,3375 & 4,0913 \\
\hline \multirow[t]{2}{*}{ preferencial } & $\mathrm{N}$ & 16 & 16 & 16 & 16 & 16 & 16 \\
\hline & $\begin{array}{l}\text { Desviación } \\
\text { estándar }\end{array}$ & ,48493 & ,54160 & ,77711 & 65749 & 63127 & ,44663 \\
\hline \multirow[t]{3}{*}{$3 \mathrm{~d}$ general } & Media & 4,1071 & 3,8286 & 3,9107 & 4,0357 & 4,3714 & 4,0507 \\
\hline & $\mathrm{N}$ & 14 & 14 & 14 & 14 & 14 & 14 \\
\hline & $\begin{array}{l}\text { Desviación } \\
\text { estándar }\end{array}$ & ,65570 & ,62687 & ,55994 & ,59531 & 43576 & ,42866 \\
\hline $3 d$ & Media & 4,4744 & 4,0308 & 3,6731 & 4,2115 & 4,5077 & 4,1795 \\
\hline \multirow[t]{2}{*}{ preferencial } & $\mathrm{N}$ & 13 & 13 & 13 & 13 & 13 & 13 \\
\hline & $\begin{array}{l}\text { Desviación } \\
\text { estándar }\end{array}$ & ,46942 & ,55884 & ,77315 & ,41890 & 34269 & 29050 \\
\hline \multirow[t]{3}{*}{$4 d$} & Media & 4,2000 & 3,8200 & 3,9250 & 4,2500 & 4,2800 & 4,0950 \\
\hline & $\mathrm{N}$ & 10 & 10 & 10 & 10 & 10 & 10 \\
\hline & $\begin{array}{l}\text { Desviación } \\
\text { estándar }\end{array}$ & ,62138 & ,64256 & ,42573 & ,70711 & ,35528 & ,37411 \\
\hline \multirow[t]{3}{*}{ Vip } & Media & 4,7783 & 4,0240 & 4,0490 & 4,5000 & 4,4267 & 4,3556 \\
\hline & $\mathrm{N}$ & 15 & 15 & 15 & 15 & 15 & 15 \\
\hline & $\begin{array}{l}\text { Desviación } \\
\text { estándar }\end{array}$ & ,31693 & 28195 & ,62830 & ,50885 & 41312 & 29507 \\
\hline \multirow[t]{3}{*}{ Total } & Media & 4,3969 & 3,9495 & 3,8599 & 4,2381 & 4,3857 & 4,1660 \\
\hline & $\mathrm{N}$ & 84 & 84 & 84 & 84 & 84 & 84 \\
\hline & $\begin{array}{l}\text { Desviación } \\
\text { estándar }\end{array}$ & ,55241 & ,57401 & 68142 & ,59731 & ,51651 & ,42781 \\
\hline
\end{tabular}

Fuente: Propia a partir de los resultados obtenidos mediante SPSS.

Por su parte, la capacidad de respuesta está, a excepción de la sala VIP, por debajo de los niveles esperados. Normalmente es así, toda vez que las demoras en cola de cafetería son traducidas como "lentitud de servicio". La posición es congruente con lo observado y valida que en la sala VIP no se tenga esta percepción en tanto que en esta sala existe servicio 
al puesto. Esto va de la mano con la dimensión confiabilidad registrada en el modelo ServQual usado y en Torres (2010).

\section{Resultados del experimento}

\section{Prueba de hipótesis}

El procedimiento que sigue se utiliza para aceptar o rechazar la hipótesis nula. En términos generales, esta hipótesis afirma que las variables "día de la película" y "tipo de sala" no son la causa de la percepción que tiene el estudiante universitario de la calidad del servicio de cine, tanto para cada una de sus características como para el servicio en general. Este procedimiento consta de seis pasos y se hace para todas las hipótesis; en las siguientes tablas (Tabla 5, Tabla 6 y Tabla 7) se presentan los cálculos estadísticos y la decisión estadística.

\section{Tabla 5}

Hipótesis nula para el factor día de la semana

\begin{tabular}{lc}
\hline \multicolumn{1}{c}{ Componente } & Hipótesis nula \\
\hline Elementos tangibles & $H_{0}=\mu_{1}=\mu_{2}=\mu_{3}$ \\
Confiabilidad & $H_{0}=\mu_{1}=\mu_{2}=\mu_{3}$ \\
Capacidad de respuesta & $H_{0}=\mu_{1}=\mu_{2}=\mu_{3}$ \\
Garantía & $H_{0}=\mu_{1}=\mu_{2}=\mu_{3}$ \\
Empatía & $H_{0}=\mu_{1}=\mu_{2}=\mu_{3}$ \\
Calidad de servicio General & $H_{0}=\mu_{1}=\mu_{2}=\mu_{3}$
\end{tabular}

Fuente: Autores.

Donde:

$\mu 1=$ Lunes-martes-jueves

$\mu 2=$ Miércoles

$\mu 3=$ Fin de semana

Se entiende que si no existe causalidad los promedios obtenidos serán iguales.

\section{Tratamiento "tipo de sala"}

Tabla 6

\section{Hipótesis nula para el factor tipo de sala}

\begin{tabular}{lc}
\hline \multicolumn{1}{c}{ Componente } & Hipótesis nula \\
\hline Elementos tangibles & $H_{0}=\mu_{1}=\mu_{2}=\mu_{3}=\mu_{4}=\mu_{5}=\mu_{6}$ \\
Confiabilidad & $H_{0}=\mu_{1}=\mu_{2}=\mu_{3}=\mu_{4}=\mu_{5}=\mu_{6}$ \\
Capacidad de respuesta & $H_{0}=\mu_{1}=\mu_{2}=\mu_{3}=\mu_{4}=\mu_{5}=\mu_{6}$ \\
Garantía & $H_{0}=\mu_{1}=\mu_{2}=\mu_{3}=\mu_{4}=\mu_{5}=\mu_{6}$ \\
Empatía & $H_{0}=\mu_{1}=\mu_{2}=\mu_{3}=\mu_{4}=\mu_{5}=\mu_{6}$ \\
Calidad de servicio General & $H_{0}=\mu_{1}=\mu_{2}=\mu_{3}=\mu_{4}=\mu_{5}=\mu_{6}$ \\
\hline
\end{tabular}

Fuente: Autores.
Donde:

$$
\begin{aligned}
& \mu 1=\text { Sala } 2 D \text { General } \\
& \mu 2=\text { Sala } 2 D \text { Preferencial } \\
& \mu 3=\text { Sala 3D General } \\
& \mu 4=\text { Sala 3D General } \\
& \mu 5=\text { Sala 4D } \\
& \mu 6=\text { Sala VIP }
\end{aligned}
$$

Se entiende que si no existe causalidad los promedios obtenidos serán iguales.

\section{Tratamiento "día de la semana y tipo de sala (interacción)"}

\section{Tabla7}

Hipótesis nula para el factor tipo de sala

\begin{tabular}{lc}
\hline \multicolumn{1}{c}{ Componente } & Hipótesis nula \\
\hline Elementos tangibles & $\alpha \beta=0$ \\
Confiabilidad & $\alpha \beta=0$ \\
Capacidad de respuesta & $\alpha \beta=0$ \\
Garantía & $\alpha \beta=0$ \\
Empatía & $\alpha \beta=0$ \\
Calidad de servicio General & $\alpha \beta=0$ \\
\hline
\end{tabular}

Fuente: Autores.

Donde:

a=El efecto de la variable "día de la película" $\beta=$ El efecto del tipo de sala $a \beta=$ Efecto de interacción

Se entiende que no existe causalidad si el producto de los efectos es igual a cero.

\section{Nivel de significancia:}

El error de tipo I para las tres pruebas se asume en [5\%].

\section{Estadístico de prueba:}

En los modelos experimentales factores fijos y diseño factorial, la prueba estadística por utilizar es el análisis de varianza (Anova).

\section{Regla de decisión}

Para todas las hipótesis se rechaza la hipótesis nula, si y solo si el estadístico Anova obtenido en el experimento es menor de $5 \%$, de lo contrario se acepta. 


\section{Cálculos estadísticos}

Los cálculos estadísticos se hacen mediante el programa SPSS versión 24. A continuación, se muestran las tres tablas (Tabla 8, Tabla 9 y Tabla 10) de análisis de varianza obtenidos.

\section{Tabla8}

Anova para la variable día de la película

\begin{tabular}{lcccc}
\hline $\begin{array}{c}\text { Elementos de la calidad } \\
\text { del servicio }\end{array}$ & $\begin{array}{c}\text { Suma de cuadrados } \\
\text { entre grupos }\end{array}$ & $\begin{array}{c}\text { Suma de } \\
\text { cuadrados dentro } \\
\text { de grupos }\end{array}$ & $\begin{array}{c}\text { Valor F de la } \\
\text { muestra }\end{array}$ & Valor sig. \\
\hline Elementos tangibles & 0,296 & 25,033 & 0,479 & 0,621 \\
Confiabilidad & 0,505 & 26,843 & 0,762 & 0,470 \\
Capacidad de respuesta & 1,609 & 36,931 & 1,765 & 0,178 \\
Garantía & 0,902 & 28,711 & 1,273 & 0,286 \\
Empatía & 0,337 & 21,806 & 0,626 & 0,537 \\
Servicio en general & 0,218 & 14,972 & 0,591 & 0,556 \\
\hline
\end{tabular}

Fuente: Elaboración propia a partir de los resultados obtenidos mediante SPSS. Nota: Decisión estadística para la variable "día de la película".

En la Tabla 8 el Anova para la variable independiente "día de la película", los valores $\mathrm{F}$ y sig. son los obtenidos en la muestra y cualquiera de ellos se utiliza para la aceptar o no la hipótesis nula. En este estudio solo se utiliza el valor sig. Para la variable independiente "día de la película", todos los valores sig. están por encima del valor de significancia (5\%), indicando que no existe suficiente evidencia para afirmar que el "día de la película" es la causa de la percepción que tiene el estudiante universitario sobre la calidad tanto en cada uno de los elementos como en la calidad del servicio en general.

\section{Decisión estadística para la variable "tipo de sala"}

En la Tabla 9, el Anova indica, inicialmente, que sí existe evidencia para para afirmar que la variable "tipo de sala" es la causa de la percepción que tiene el estudiante de la calidad de los "elementos tangibles" del servicio de cine. Resultado interesante, porque los empresarios del cine pueden manipular todo lo que tiene que ver con las sillas, la limpieza de las instalaciones, la presentación de los empleados, y la amplitud, comodidad y decoración de las instalaciones. Estos elementos son claves para que los usuarios perciban una mejor calidad en el servicio.

\section{Tabla 9}

\section{Anova para la variable tipo sala}

\begin{tabular}{lcccc}
\hline $\begin{array}{c}\text { Elementos de la calidad } \\
\text { del servicio }\end{array}$ & $\begin{array}{c}\text { Suma de } \\
\text { cuadrados entre } \\
\text { grupos }\end{array}$ & $\begin{array}{c}\text { Suma de cuadrados } \\
\text { dentro de grupos }\end{array}$ & $\begin{array}{c}\text { Valor F de la } \\
\text { muestra }\end{array}$ & Valor sig. \\
\hline Elementos tangibles & 4,034 & 21,294 & 2,955 & 0,017 \\
Confiabilidad & 0,742 & 26,605 & 0,435 & 0,823 \\
Capacidad de respuesta & 1,215 & 37,325 & 0,508 & 0,769 \\
Garantía & 1,732 & 27,881 & 0,969 & 0,442 \\
Empatía & 0,372 & 21,771 & 0,267 & 0,930 \\
Servicio en general & 0,883 & 14,307 & 0,963 & 0,446 \\
\hline
\end{tabular}

Fuente: Elaboración propia a partir de los resultados obtenidos mediante SPSS.

Una lectura adicional es que la sala, en sí misma, es un producto altamente diferenciado; que la calidad percibida de las relaciones ambientales medidas en términos de estímulos sensoriales diferentes o más comodidad, es mayor, y forman parte de una ventaja competitiva sostenible versus otras tecnologías adyacentes. 
Con relación a los otros componentes de la calidad del servicio se encontró que no existe evidencia para afirmar que la variable "tipo de sala" sea la causa de la percepción que tiene el estudiante sobre la calidad de cada componente y la calidad del servicio en general.

Tabla 10

Anova para la interacción día de la película y tipo de sala

\begin{tabular}{|c|c|c|c|c|c|c|}
\hline & $\begin{array}{c}\text { Elementos } \\
\text { tangibles }\end{array}$ & Confiabilidad & $\begin{array}{l}\text { Capacidad de } \\
\text { respuesta }\end{array}$ & Garantía & Empatía & $\begin{array}{c}\text { Servicio } \\
\text { en } \\
\text { general }\end{array}$ \\
\hline Modelo corregido & $10,490 a$ & $3,962 a$ & $12,087 a$ & $8,601 a$ & $4,508 a$ & $3,353 a$ \\
\hline Intersección & 1349,183 & 1083 & 989,061 & 1248,340 & 1325,861 & 1194,865 \\
\hline Día & 0,131 & 0,520 & 2,192 & 1,182 & 0,116 & 0,171 \\
\hline Tipo & 3,972 & 0,584 & 3,079 & 1,895 & 0,310 & 0,811 \\
\hline Día * tipo & 5,993 & 2,738 & 8,967 & 5,919 & 3,775 & 2,179 \\
\hline Valor $\mathrm{f}$ modelo & 2,745 & 0,658 & 1,774 & 1,589 & 0,992 & 1,100 \\
\hline Valor sig modelo & 0,002 & 0,832 & 0,051 & 0,093 & 0,477 & 0,373 \\
\hline Valor f intersección & 6001,234 & 3056,561 & 2467,693 & 3921,021 & 4962,206 & 6661,951 \\
\hline Valor sig intersección & 0,000 & 0,000 & 0,000 & 0,000 & 0,000 & 0,000 \\
\hline Valor F día & 0,292 & 0,734 & 2,734 & 1,857 & 0,217 & 0,476 \\
\hline Valor sig dia & 0,748 & 0,484 & 0,072 & 0,164 & 0,805 & 0,624 \\
\hline Valor $\mathrm{ftipo}$ & 3,534 & 0,330 & 1,536 & 1,190 & 0,232 & 0,905 \\
\hline Valor sig tipo & 0,007 & 0,893 & 0,191 & 0,324 & 0,947 & 0,483 \\
\hline Valor F interacción & 2,666 & 0,773 & 2,237 & 1,859 & 1,413 & 1,215 \\
\hline Valor sig interacción & 0,008 & 0,654 & 0,026 & 0,067 & 0,194 & 0,298 \\
\hline
\end{tabular}

Fuente: Elaboración propia a partir de los resultados obtenidos mediante SPSS.

\section{Decisión estadística para la interacción de las variables "día de la película" y"tipo de sala"}

La Tabla 10 recoge evidencia estadísticamente significativa que permite demostrar que el efecto de interacción "día de la película" y "tipo de sala" sí contribuye en la percepción de calidad de dos elementos: tangibilidad y capacidad de respuesta. Los otros elementos no mostraron evidencias significativas.

\section{Comentarios}

Concluyendo, es menester destacar el papel que tienen los elementos tangibles y la capacidad de respuesta en la calidad percibida para los factores "tipo de sala" y"día de la película". Así las cosas, si las salas desean mantener un nivel de respuesta percibida alto deben cubrir los elementos tangibles de cine y sala propuestos por Torres (2010), que incluyen condiciones ambientales (aroma, música ambiental, iluminación), al tiempo con características inherentes a la sala como temperatura, sonido y comodidad de butacas entre otros. Las características anteriores influyen de manera explícita en la sensación y percepción del cliente, toda vez que el espectáculo de cine que se propone es altamente sensorial, definido por el lenguaje visogénico -entendido como las sensaciones creadas por las combinaciones de sonidos e imágenes, combinaciones que sobrepasan estos dos campos y donde los efectos de luz y sonido juegan un papel preponderante (Chion, 2013)-. Se deben implementar estrategias desde la disciplina y la praxis que generen mejores resultados.

De otra parte, la calidad de respuesta se entiende como todo esfuerzo del oferente para minimizar tiempos de espera. Aquí el papel de la tecnología como propiciador de acceso juega un papel importante (compra de boletas por Internet, por ejemplo); además, se sugiere disminuir los tiempos de espera en la fila mediante la intervención de personal capacitado para solventar en menor tiempo la demanda de alimentos, lo cual redundará en una mejor experiencia percibida (Ibarra, Casas \& Partida, 2011).

\section{Referencias}

Anzures,F.(2013).Elconsumidoreselmedio.Bogotá:Panamericana. Artopoulos, A. (2011) La sociedad de las cuatro pantallas. Una mirada latinoamericana. Buenos Aires: Fundación Telefónica.

Ato, M., López, J. \& Benavente, A. (2013). Un sistema de clasificación de los diseños de investigación en psicología. Anales de psicología, 29(3), 1038-1059.

Barberà, O. \& Valdés, P. (1996). El trabajo práctico en la 
enseñanza de las ciencias: una revisión. Enseñanza de las ciencias: revista de investigación y experiencias didácticas, 14(3), 365-379.

Berdugo, C., Barbosa, R. \& Prada, L. (2016). Variables relevantes para la medición de la calidad percibida del servicio bancario. Dyna, 83(197), 212.

Chacín, F. (2000). Diseño y análisis de experimentos para generar superficies de respuesta. Maracay Venezuela: Facultad de Agronomía Universidad Central de Venezuela.

Chion, M. (2013). Aspectos de lo sensorial en el cine actual. Toma uno, (2), 13-19.

Díaz, V. (2009). Errores estadísticos frecuentes al comparar dos poblaciones independientes. Revista chilena de nutrición, 36(4), 1136-1138.

Duque, E. (2005). Revisión del concepto de calidad del servicio y sus modelos de medición. Revista Innovar Journal Revista de Ciencias Administrativas y Sociales, 15(25), 64-80.

Folgueiras, P., Luna, E. \& Puig, G. (2013). Aprendizaje y servicio: estudio del grado de satisfacción de estudiantes universitarios. Revista de educación, 362, 159-185.

García, D. \& Amante, B. (2006). Algunas experiencias de aplicación del aprendizaje cooperativo y del aprendizaje basado en proyectos. En I Jornadas de Innovación Educativa. Escuela Politécnica Superior de Zamora.

Giraldo, M. (2016). Creación de soluciones: administrando propuestas de valor en el mercado. En Giraldo, M. Juliao, D., et al (2016). Gerencia de Marketing (pp. 4055). Barranquilla, Colombia: Universidad del Norte.

Grönroos, C. (1984). A service quality model and its marketing implications. European Journal of marketing, 18(4), 36-44.

Grönroos, C., Storbacka, K. \& Strandvik, T. (1994). Managing customer relationships for profit: the dynamics of relationship quality. International journal of service industry management, 5(5), 21-38.

Gutiérrez, H. \& De la Vara, R. (2004). Análisis y diseño de experimentos. México: McGraw-Hill.

Ibarra, L., Casas, E. \& Partida, A (2011). Método ServQual aplicado a las salas de cine, Cinemark y Cinépolis: un análisis comparativo sobre la calidad en el servicio, caso Hermosillo, Sonora. RIDE Revista Iberoamericana para la Investigación y el Desarrollo Educativo, 2(3).

Jaramillo, L., Reina, M. \& Zuleta, L. (2003). Impacto del sector cinematográfico sobre la economía colombiana: situación actual y perspectivas. Colección economía y cultura: Fedesarrollo.

Losada, M. \& Rodríguez, A. (2007). Calidad del servicio de salud: una revisión a la literatura desde la perspectiva del marketing. Cuadernos de Administración, 20(34), 237-258.

Machado, E., Montes, N. \& Mena, A. (2008). El desarrollo de habilidades investigativas como objetivo educativo en las condiciones de la universalización de la educación superior. Pedagogía universitaria, 13(1).
Malhotra, N. K. (2007). Investigación de Mercados: un enfoque aplicado (6a. ed.): Pearson educación.

Martínez, V. \& Tordera, N. (1995). Comparación de modelos causales sobre satisfacción del usuario. Estudios sobre consumo, 34, 13-22.

Marzo, J., Martínez, V., Ramos, J. \& Peiró, J. (2002). La satisfacción del usuario desde el modelo de la confirmación de expectativas: respuesta a algunos interrogantes. Psicothema, 14(4).

McDaniel, C. \& Gates, R. (2015). Investigación de mercados (10a. ed.). México: Cengage.

Mejías, A. (2005). Validación de un instrumento para medir la calidad de servicio en programas de estudios universitarios. Ingeniería Industrial, 26(2), 8.

Mesa, J. (2005). Del marketing de servicios al marketing relacional. Revista colombiana de marketing, 4(6).

Moguillansky, M. (2016). El nuevo cine de espectáculo como estrategia comercial. Un análisis de las tendencias del consumo de cine en salas. Intercom: Revista Brasileira de Ciências da Comunicação, 39(3), 167-183.

Molla, A., Eiglier, P.\& Langeard, E. (1989). Servucción: McGraw Hill. Nagle, T., Tagle, R. \& Holden, R. (2002). Estrategia y tácticas de precios: una guía para tomar decisiones rentables: Prentice-Hall.

Parasuraman, A., Berry, L. L., \& Zeithaml, V. A. (1991). Refinement and reassessment of the SERVQUAL scale. Journal of retailing, 67(4), 420.

Parasuraman, A., Berry, L., \& Zeithaml, V. (1992). Refinement and reassessment of the Servqual scale. Journal of retailing, 67(4), 420.

Parasuraman, A., Zeithaml, V. \& Berry, L. (1985). A Conceptual Model of Service Quality and its Implications for Future Research. Journal of Marketing, 49(4), 41-50.

Parasuraman, A., Zeithaml, V. \& Berry, L. (1988). Servqual: A multiple-item scale for measuring consumer perceptions of Service Quality.J Retailing, 6,12-37.

Parasuraman, A., Zeithaml, V. \& Berry, L. (1993). Calidad total en la gestión de servicios. Madrid: Díaz de Santos.

Pascual, J., García, J. \&Frías, M. (1996). El diseñoy la investigación experimental en Psicología. Valencia, España.

Restrepo, N. (2007). Estrategia de precios: un enfoque de mercadeo para los negocios. Universidad Eafit.

Saren, M. (2006). Marketing graffiti: the view from the street: Routledge.

Sureshchandar, G., Rajendran, C. \& Kamalanabhan, T. (2001). Customer perceptions of service quality: A critique. Total quality management, 12(1), 111-124.

Torres, E. (2010). Escala propuesta para medir la calidad del servicio de los cines. Innovar, 20(36), 157-171. 